\title{
Factor Copula Models and Their Application in Studying the Dependence of the Exchange Rate Returns
}

\author{
Hanyue Zhang (Corresponding Author) \\ Department of Economics, University of Toronto \\ 150 St George St., Toronto, ON M5S 3G7, Canada \\ Tel: 1-416-829-0288Ｅ-mail: shinya.zhang@mail.utoronto.ca
}

Feng Jiao

Desautels Faculty of Management, McGill University

1001 Rue Sherbrook Ouest, Montreal, QC, H3A 1G5, Canada

Tel: 1-438-998-1998 E-mail: feng.jiao@mail.mcgill.ca

Received: September 19, 2011

Accepted: December 8, 2011

Published: February 1, 2012

doi:10.5539/ibr.v5n2p3

URL: http://dx.doi.org/10.5539/ibr.v5n2p3

\begin{abstract}
This paper applies multivariate factor copula modeling methods to study the dependence relationships of exchange rates. We found that conditional on the common factors, the dependence among the chosen currencies is weakly asymmetric, and the two-factor Gaussian copula modeling hypothesis is more appropriate.
\end{abstract}

Keywords: Exchange rate, Gaussian Copula, Gumbel Copula, Factor analysis, Principle component analysis

\section{Introduction}

The global financial crisis that began in 2007 has led to extreme volatility in the foreign exchange market. The movement of the Canadian dollar has a great impact on the Canadian people's living standards and domestic economy. The Canadian government needs to predict the position of the Canadian dollar in global markets quickly in order to stabilize the economy and minimize the risk of holding different types of currencies. Corporations need to know the same information in order to minimize the risk of holding certain currencies when they are conducting international business. Individual investors also increasingly need to know the same information to plan household budgets and change consumption habits to accommodate the rising and falling currency. It is therefore necessary, and important, to investigate the relationships among the Canadian dollar and other currencies, especially during volatile periods. This research will examine these relationships during economic booms and recessions.

One of the simplest and most commonly used approaches to study dependence among the exchange rates is to calculate the linear correlation. Unfortunately, this approach is problematic. First, it is a widely recognized that the return data usually exhibit typical asymmetric distributions instead of a symmetric normal distribution. Second, the linear correlation cannot measure the dependence structure (i.e., how the markets depend on each other). The exchange rate returns' correlations may increase more when the economy is in recession than when the economy is booming. The linear correlation coefficient may underestimate the dependence of the financial exchange markets during the crisis since it does not capture the excess co-movements when the markets move downwards (Patton, 2006; Patton, 2009). Therefore, the linear correlation coefficients are inappropriate. Fortunately, an alternative approach, copula modelling, can overcome the limitations of the linear correlation coefficients technique. Therefore, as suggested by $\mathrm{Hu}$ (2006), this paper applies copulas to study both dependence degree and dependence structure among the exchange rate returns.

A copula is a cumulative distribution function connecting multivariate marginal distributions in a specified form. Usually, researchers can obtain information about the distributions of the marginal functions, but it is difficult to accurately specify joint distributions. Copula modelling offers an important improvement in this respect: for example, in a bivariate case, when both marginals are Gaussian and the copula has a Gaussian distribution, the joint distribution generated will be Gaussian as well. However, even if the marginals are not Gaussian, a copula approach can still be used to generate a joint distribution (Hu, 2006; Longin and Solnik, 2001). 
Besides modelling the joint distribution with copulas, this paper applies factor analysis to identify the common factors and construct two-factor copula models to study the dependence among the exchange rate returns. The common factors play an important role in determining the joint dependence among the returns. The common factors can be oil price, global consumer confidence index, or the combination of these factors. When the returns on these factors change, all exchange rate returns will be affected to different degrees depending on how much their representative economies are tied with these factors, and this contributes to the asymmetric joint distributions of the chosen returns. To focus solely on the interactions of the dependence among the currencies themselves, the copula models conditional on the common factors (also named as factor copula models in this paper) are more preferable. The common factors, also known as principal components in this study, can be found by using a principal component analysis technique, which will be described in further details in the methodology section.

In contrast to the existing literature that focuses mainly on the bivariate studies, this paper focuses on Gaussian, Clayton, and Gumbel copula modelling in the multivariate case. The aim of this paper is to apply factor copula models to study dependence relationships among daily returns of these chosen exchange rates. In addition, this paper confirms if there is asymmetric dependence among the chosen exchange rate returns or not, and provides suggestions on identifying the appropriate copula for the chosen exchange rates.

The structure of the remainder of this paper is organized as follows: Section 2 presents a review of relevant literature; Section 3 reviews the history, basic concepts, special forms and properties of copulas selected for this study, and a brief introduction on principal component analysis; Section 4 describes the data used for empirical investigation and estimation procedures, and finally, the last section concludes the study and contains suggestions for future research.

\section{Literature Review}

The first few scholars thoroughly introduced one-factor Gaussian copulas, one-factor mean variance Gaussian mixtures, and one-factor Archimedean copulas. The factor approach helped achieve the goal of data reduction by locating the common factor variables affecting the selected data series. Conditional on these factors, the copulas can better assist in identifying the dependence relationships and model the chosen financial data. Furthermore, Anderson and Sidenius (2004) extended the standard Gaussian copula model to two new models to study the portfolio default loss. However, the existing studies focus mainly on the one-factor copulas. In contrast, this research focuses on using two-factor copulas, and the selected copula models are Gaussian, Gumbel, and Clayton copulas.

The attention of exchange rates has gradually shifted towards using copula tools to verify if the distributions of these exchange rates are asymmetric and to model their dependence. These studies will in turn assist the risk management of the international investment portfolio that can be significantly affected by the fluctuations in currencies. Hurd, Salmon, and Schleicher (2007) applied copulas to construct bivariate foreign exchange distributions with a focus on the application of the Sterling Exchange Rate Index. Built on their study, Patton (2006) extended the copulas conditional on variables or common factors found through the factor approach, and constructed flexible models of the conditional dependence structure of the mark-dollar and yen-dollar exchange rates. Notably, the research that has been done focuses on applying bivariate copulas only for the dependence between two exchange rates. This research contributes to the existing literature by focusing on multivariate factor copula models to study four exchange rates: CAN/U.S., pound/U.S., yen/U.S., and euro/U.S. dollar.

\section{Methodology}

\subsection{Definitions and Properties of Copula Functions}

Before we proceed to the foundational theorem for copulas due to Sklar (1959), we begin with the formal definition of "copula:

Definition 1: A two-dimensional copula is a function C: $[0,1]^{2} \rightarrow[0,1]$ which has the following properties:

(1) $C(u, v)$ is increasing in $\mathrm{u}$ and $\mathrm{v}$;

(2) $C(0, v)=C(u, 0)=0, C(1, v)=v, C(u, 1)=u$;

(3) For every $\mathrm{u}_{1}, \mathrm{u}_{2}, \mathrm{v}_{1}, \mathrm{v}_{2} \in[0,1]$ such that $\mathrm{u}_{1}<\mathrm{u}_{2}$ and $\mathrm{v}_{1}<\mathrm{v}_{2}$, we have $C\left(\mathrm{u}_{2}, \mathrm{v}_{2}\right)-C\left(\mathrm{u}_{2}, \mathrm{v}_{1}\right)-C\left(\mathrm{u}_{1}, \mathrm{v}_{2}\right)+C$ $\left(\mathrm{u}_{1}, \mathrm{v}_{1}\right) \geq 0$

The first property indicates that the joint distribution function increases when allowing one variable to increase while keeping the other one constant. The second property ensures that the copula function is zero when the probability of one variable is zero. Additionally, the joint probability is determined by the marginal probability that is not equal to one.

To further introduce the formal definition of copulas and show how copulas are used to restore joint distributions for marginals, now we state Sklar's Theorem. 
Sklar's Theorem in n-dimensions (1959): Let $\mathrm{H}$ be an n-dimensional distribution function with marginal $\mathrm{F}_{\mathrm{i}}(\cdot)$ with $\mathrm{i}=1, \ldots, \mathrm{n}$. Then, there exists a copula $\mathrm{C}$ such that for random variables $\mathrm{X}_{\mathrm{i}}$ we have

$$
H\left(x_{1}, x_{2}, x_{3}, \ldots, x_{n}\right)=C\left(F_{1}\left(x_{1}\right), F_{2}\left(x_{2}\right), \ldots, F_{n}\left(x_{n}\right)\right) .
$$

If $\mathrm{F}_{\mathrm{i}}(\cdot)$ are continuous for all $\mathrm{i}=1, \ldots, \mathrm{n}$, then $\mathrm{C}$ is unique. Conversely, if $\mathrm{F}_{\mathrm{i}}(\cdot)$ are marginals or CDFs and $\mathrm{C}$ is a copula with a range of $[0,1]^{\mathrm{n}}$, then the function $\mathrm{H}$ defined in (1) is a joint distribution function with one-dimensional marginals $\mathrm{F}_{\mathrm{i}}(\cdot)$. Notably, when $\mathrm{H}$ is continuous, the unique $\mathrm{C}$ will be

$$
C\left(u_{1}, u_{2}, \ldots, u_{n}\right)=H\left(F_{l}^{-1}\left(u_{1}\right), F_{l}^{2}\left(u_{2}\right), \ldots, F_{n}^{-n}\left(u_{n}\right)\right) .
$$

Applying a copula function to restore the bivariate distribution has several advantages. First, it provides flexibility in the model specification by separating the specifications of the marginals from those of the copula. In this way, it is possible to construct a complex non-Gaussian joint distribution. Second, a copula is a powerful technique because it directly models the dependence between the marginal distribution functions.

\subsection{Some Common Copulas}

After giving a general definition for copulas, in this section, we present several important copulas and their properties. We first define the product copula, which is the simplest copula function. Then we define the Gaussian copula, which is the basic and most commonly used copula. After this, we present an important class of copula functions: Archimedean copulas. The copulas that fall in this class can be stated directly and usually have a simple closed form expression. In addition, these copulas are popular because they can be easily derived and can capture a wide range of dependence structures.

\subsubsection{Gaussian Copula}

As Schmidt (2006) outlined in his work, Gaussian copulas are an extension from the multivariate normal distribution. Let us assume that $X_{1}$ and $X_{2}$ are normally distributed and they are also jointly normal. Then we can use a linear correlation to fully describe their dependence structure. Let $\Phi_{\Sigma}$ be the CDF of multidimensional normal distribution, the multivariate Gaussian copula for a correlation matrix $\Sigma$ is given by

$$
C_{\Sigma}^{G}(u)=\Phi_{\Sigma}\left(\Phi^{-1}\left(u_{1}\right), \ldots, \Phi^{-1}\left(u_{n}\right) ; \Sigma\right)
$$

Note that the linear correlation is a good and efficient measure of the dependence relationships in this case. The equivalent way of expressing Gaussian independence is zero correlation. Positive (or negative) sign of $\theta$ reveals a positive (or negative) linear dependence relationship among the variables.

\subsubsection{Clayton Copula}

As mentioned by Trivedi and Zimmer (2005), with $\theta \in[-1, \infty]\{0\}$ and $\mathrm{n} \geq 2$, the $n$ dimensions Clayton Copula takes the form

$$
C_{\theta}^{n}(u)=\left(\mathrm{u}_{1}^{-\theta}+\mathrm{u}_{2}^{-\theta}+\cdots+\mathrm{u}_{\mathrm{n}}^{-\theta}-n+1\right)^{-\frac{1}{\theta}}
$$

\subsubsection{Gumbel Copula.}

As mentioned by Trivedi and Zimmer (2005), if $\theta \geq 1$ and any $n \geq 2$, we have $n$ dimensions Gumbel copulas as

$$
C_{\theta}^{n}(u)=\exp \left(-\left[\left(-\ln u_{1}\right)^{\theta}+\left(-\ln u_{2}\right)^{\theta}+\cdots+\left(-\ln u_{n}\right)^{\theta}\right]^{-1 / \theta}\right)
$$

\subsection{Measuring Dependence}

\subsubsection{Linear Correlation}

In statistics and economics literature, the most familiar concept in studying dependence is the correlation coefficient between two random variables. The correlation coefficient $\rho$ is a well studied measure of linear dependence and it is symmetric. Note that the correlation measure is invariant under linear transformations of the variables, but it does not hold for general transformations. Finally, $\rho=0$ implies independence for bivariate normal distributed random variables, but it does not hold in general.

\subsubsection{Rank Correlation}

Unlike linear correlation, rank correlation is an alternative measure of nonlinear dependence relationships among variables with non-Gaussian marginals. As implied by its name, rank correlation concentrates on modeling the rankings of given observed data rather than on the actual values of the data themselves. Given by Trivedi and Zimmer (2005), there are two well-established measures of rank correlation, Spearman's rho and Kendall's tau, which provide a way to fit copulas to data. 
Both Spearman's rho, $\rho_{S}(x, y)$, and Kendall's tau, $\rho_{\tau}(x, y)$, have the following four properties: first, they are symmetric; second, they are bounded by $(-1,1)$, and their lower and upper bounds on this inequality measure perfect negative and positive linear dependence; third, they are equal to zero when the random variables are independent from each other; finally, they are co- and counter- monotonic. The expressions in terms of copulas for the Rank correlations are as follows:

$$
\rho_{S}(x, y)=12 \iint_{00}^{11}\left\{C\left(u_{1}, u_{2}\right)-u_{1} u_{2}\right\} d u_{1} d u_{2}
$$

and

$$
\rho_{\tau}(x, y)=4 \int_{00}^{11} C\left(u_{1}, u_{2}\right) d C\left(u_{1}, u_{2}\right)-1
$$

According to Nelson (1999), Kendall's tau and Spearman's rho are equivalent with the same underlying assumptions, but they usually have similar but different magnitudes. These two methods can verify the changes in the dependence relationships in different subsamples since the directions of the changes are usually the same under both methods. In this paper, our results include the estimates of the Kendall's tau, the Spearman's rho, as well as the tail indices (i.e., tail dependence), which is introduced in the following section.

\subsubsection{Tail Dependence}

$\mathrm{n}$ this subsection, we introduce the concept of tail dependence, which is applied to measure the dependence between the extreme values of random variables, for copula models. We refer to extreme co-movement relationships as concepts of concordance and discordance. Basically, according to Trivedi and Zimmer (2005), concordance means that there is a dependent relationship between large values of two random variables, and discordance means that there is a dependent relationship between large values of one random variable with small values of another.

To find the tail dependence with our copula functions, the following calculations give upper $\left(\lambda_{U}\right)$ and lower tail $\left(\lambda_{L}\right)$ dependence:

$$
\lambda_{U}=\lim _{m \rightarrow 1^{-}} P\left(x_{2}>F_{2}^{-1}(m) \mid x_{1}>F_{1}^{-1}(m)\right)=\lim _{m \rightarrow 1^{-}} \frac{C^{S}(m \cdot m)}{1-m}
$$

and

$$
\lambda_{L}=\lim _{m \rightarrow 0^{+}} P\left(x_{2} \leq F_{2}^{-1}(m) \mid x_{1} \leq F_{1}^{-1}(m)\right)=\lim _{m \rightarrow 0^{+}} \frac{C^{S}(m \cdot m)}{1-m}
$$

The left and right tail dependences of the Gaussian Copula are identical. Hence, the Gaussian Copula is a good choice for modeling between two variables when there is no strong tail dependence. Or, in the context of exchange rate returns, when two rates are not strongly correlated at low (or high) values but less correlated at high (or low) values, the Gaussian copula is an appropriate modeling choice.

The Clayton Copula has strong positive left tail dependence and relatively weak right tail dependence. In other words, it models extreme negative co-movements. The Clayton Copula is a good choice for modeling between two variables when their left tail dependence is strong. Or, in the context of exchange rate returns, when two returns are strongly correlated at low values but less correlated at high values, the Clayton copula is an appropriate modeling choice.

The Gumbel copula has strong positive right tail dependence and relatively weak left tail dependence. In other words, it does not tolerate extreme negative co-movements. In the context of exchange rate returns, when correlation between two returns is strong in the right tail of the joint distribution, the Gumbel copula is an appropriate choice.

Therefore, if we can estimate the dependence parameter in each copula function, we can easily calculate out the values of rank correlations, which include both the Kendall's tau and the Spearman's rho, and the tail dependence structure for that copula.

\section{Factor Copula Structure}

In this paper, we introduce an alternative way of approaching the problem of finding the dependence relationships between fluctuations on exchange rates by using copula models conditional on the common factors found through the factor analysis.

Factor analysis is based on the assumption that some underlying factors, which are smaller in number than the number of observed variables, are responsible for the co-variation among the observed variables. This analysis 
method is mainly used for data reduction purposes. This paper focuses on the principal component analysis method, which is the most common form of factor analysis. These principal components are able to account for most of the variance in the observed exchange rate returns.

Instead of directly applying the marginal distributions of the exchange rate returns into our copula function, we can use their marginals conditional on the common factors. The correlation coefficients of these returns depend on the estimated common factors. The ways the returns are interrelated with each other depend largely on the common factors. To focus solely on the interactions among the returns, the factor copula correlations conditional on the estimated common factors can more accurately describe the dependence relationships among multiple variables. Specifically, conditional on the common factors, the exchange rate returns will only dependent on the joint distributions of $Z_{1}, Z_{2}, \ldots, Z_{n}$, which are the unique parts for their respective exchange rate returns.

\subsection{Two factor Gaussian Copulas.}

We define $r_{i}(i=1,2, \ldots, n)$ as the returns on the exchange rates. If these returns were normally distributed, the joint distribution of them may be multivariate normal. As is well-known in the academic world, the probability distribution of financial series tends not be normal. To apply a Gaussian copula to model our data, we followed the suggestions of Hull (2009) and first transformed the returns into new variables $\mathrm{x}_{\mathrm{i}}(\mathrm{i}=1,2, \ldots, \mathrm{n})$ using $x_{i}=N^{-1}\left[Q_{i}\left(r_{i}\right)\right], \mathrm{i}=1,2, \ldots, \mathrm{n}$, where $\mathrm{N}^{-1}$ is the inverse of the cumulative normal distribution and $\mathrm{Q}_{\mathrm{i}}(\mathrm{i}=1,2, \ldots, \mathrm{n})$ are the cumulative distribution functions for respective exchange rate returns, $r_{i}(i=1,2, \ldots, n)$. In this transformation, the new variables, $\mathrm{x}_{\mathrm{i}}$, are constructed to have a standard normal distribution with mean equals to zero and standard deviation equals to one. This transformation is percentile to percentile so that the correlations among the returns can be measured by the ones among the new variables. Then introducing a Gaussian copula, we can study the copula correlations or dependence relationships among financial returns that do not have normal distribution, and separate the estimations for unconditional marginal distributions and the joint distribution.

In the two-factor model,

$$
x_{i}=\alpha_{i} F_{1}+\beta_{i} F_{2}+\sqrt{1-\alpha_{i}-\beta_{i}} Z_{i}
$$

In this equation, $F_{1}$ and $F_{2}$ are two common factors affecting defaults for all companies and $Z_{i}$ have independent standard normal distributions. The $\alpha_{i}$ and $\beta_{i}$ are constant parameters between -1 and +1 . The correlation between $\mathrm{x}_{\mathrm{i}}$ and $\mathrm{x}_{\mathrm{j}}$ is $\alpha_{i} \alpha_{j}+\beta_{i} \beta_{j}$.

Suppose that the probability that exchange rate $\mathrm{i}$ will be below a threshold of $\mathrm{m}$ is $\mathrm{Q}_{\mathrm{i}}(\mathrm{m})$. Under the Gaussian copula model, such low returns happen when $\mathrm{N}\left(\mathrm{x}_{\mathrm{i}}\right)=\mathrm{Q}_{\mathrm{i}}(\mathrm{m})$ or $\mathrm{x}_{\mathrm{i}}=\mathrm{N}^{-1}\left[\mathrm{Q}_{\mathrm{i}}(\mathrm{m})\right]$. From equation (7), this condition is

$$
Z_{i}=\frac{N^{-1}\left[Q_{i}(m)\right]-\alpha_{i} F_{1}-\beta_{i} F_{2}}{\sqrt{1-\alpha_{i}-\beta_{i}}}
$$

Conditional on the value of the factors $\mathrm{F}_{1}$ and $\mathrm{F}_{2}$, the probability of having a return lower than $\mathrm{m}$ is therefore

$$
Q_{i}(m \mid F)=N\left(\frac{N^{-1}\left[Q_{i}(m)\right]-\alpha_{i} F_{1}-\beta_{i} F_{2}}{\sqrt{1-\alpha_{i}-\beta_{i}}}\right)
$$

Therefore, setting a threshold of $\mathrm{m}$, we can find out the probability of having such disappointing returns.

\subsection{Two-Factor Archimedean Copulas}

In addition, by using the factors we found, we can extend the copula models for conditional variables. Besides one factor Gaussian copulas, Granger et al. (2006) outlined the one-factor Clayton copula model in their paper. Based on their work, we extended their model and constructed two-factor Clayton and Gumbel copulas.

The first step is to identify the common factors. The common factors are extracted from the returns without transforming them to normal variables. Instead of having a normal distribution, the common factors follow a Gamma distribution with parameter $1 / \theta$, where $\theta>0$, and with a scale parameter equal to one. Then we define

$$
X_{i}=\left(1-\frac{\ln \left(U_{i}\right)}{F}\right)^{-1 / \theta}
$$

where $\mathrm{U}_{\mathrm{i}}(\mathrm{i}=1, \ldots, \mathrm{n})$ are independent uniform random variables and they are independent from the common factors, F. Conditionally on the common factors, for Clayton copula, we have

$$
Q_{i}^{\text {Clayton }}(m \mid F)=\exp \left(V\left(1-Q_{i}(t)^{-\theta}\right)\right)
$$


and using similar logic for Gumbel copula, we have

$$
Q_{i}^{\text {Gumbel }}(m \mid F)=\exp \left(-V \log \left(Q_{i}(t){ }^{\theta}\right)\right)
$$

Using above conditional CDFs, we were able to find more accurate copula correlations or dependence relationships among the exchange rate returns excluding the common factors.

\section{Estimation Methods}

This paper's main interest is to estimate the dependence parameters in copula functions. We adopt the approach called two-stage maximum likelihood estimation method: in the first stage, the marginal distribution functions are estimated with the assumption of independence between the two random variables; in the second stage, the estimated marginal distributions are substituted into the copula function and the dependence parameter of this copula function is estimated. The marginal distributions and the dependence structure are independently estimated. Using the two-stage maximum likelihood method, we don't need to make any assumption on the marginal distributions and we can use the estimated marginal distributions, which mean that the estimated distributions are free of specification error. Therefore, this paper focuses on the two-stage maximum likelihood method.

\section{Data}

The data used in this study were from DataStream. Our research interest is to investigate the dependence among foreign exchange rates. The data of interest therefore were the daily returns of exchange rates in four different countries/regions. These selected currencies are the Canadian Dollar, British Pound, Japanese Yen, and Euro. The abbreviations used are CAD (Canadian Dollar), GBP (British Pound), JPY (Japanese Yen), and EUR (Euro).

We chose the start date of launching the Euro, which is January $2^{\text {nd }}, 2002$, as the cut-off point, and eliminated any observations before this. The sample data end with October $29^{\text {th }}, 2010$. This reduces the sample to 1,730 observations. In addition, the current financial crisis would influence the empirical results we obtained on a large scale. It is interesting to compare the pre- and post- crisis empirical results of sample observations. To achieve this goal, we used the same filtered sample data but we further separated the sample by the cut-off date of July $1^{\text {st }}, 2007$ (note: this is a rough estimation of the start date for our current crisis). The first subsample has 1,037 observations between January $2^{\text {nd }}, 2002$ and June $30^{\text {th }}, 2007$, while the second subsample has 693 observations between July $2^{\text {nd }}$, 2007 and October $29^{\text {th }}, 2010$.

\section{Empirical Results}

\subsection{Gaussian Copula with Factor Loadings}

After transforming the non-Gaussian exchange rate returns into the normal variables, we found the common factors of these variables with the factor loadings shown in Table 5a and the percentages that the principal components or common factors can explain the underlying data are exhibited in Table $5 b$.

Since the transformation is a percentile-to-percentile transformation (note: the details are described under the section of the methodology), the correlations among the exchange rate returns can be measured as the correlations among these transformed normal variables, and these calculated correlations are referred to as the copula correlations (Hull, 2008). The copula correlations in both the unconditional and two-factor Gaussian Copula models are reported in Tables 6 and 7.

Comparing the Tables 6 and 7 with Table 4, we noticed that without using copula models that can capture nonlinear relationships, there are no significant linear correlations of the CAN/US returns with the JPY/US as shown in Table 4, while using copula models, we identified significant copula correlations as shown in both Tables 6 and 7 . As we can see from both Tables 6 and 7, the copula correlations tend to exhibit a mixed result in terms of increased or decreased dependence relationships. All copula correlations between the exchange rate returns decreased significantly after the crisis occurred except the correlations of the CAN/US returns with the JPY/US and UK/US returns for the unconditional Gaussian copula models. For the two-factor copula, all copula correlations decreased significantly after the crisis occurred except the correlations of the CAN/US returns with the JPY/US and EUR/US returns. This can be explained by the relative stability of the economy and banking system in Canada compared to the ones in Japan, UK, and European.

\subsection{Clayton and Gumbel Copula with Factor Loadings}

For Clayton and Gumbel Copulas, the estimates of the common factor loadings for the exchange rate returns are exhibited in Table 8a and the percentages that the principal components or common factors can explain the underlying data are exhibited in Table 8 b.

Conditional on the common factors, the estimates for the dependence parameters, the rank correlations, and tail 
indices for both the unconditional and two-factor Clayton and Gumbel copulas are shown in Table 9.

Table 9 shows that the dependence parameters tend to decrease after the crisis occurred. In other words, after the crisis occurred, the returns on the currencies tend to be less dependent on each other, and the dependence parameters of unconditional Gumbel copula tend to be more than double the estimations of unconditional Clayton copula. This means that the dependence among the returns is asymmetric, and these returns are more negatively dependent on each other. Therefore, the models such as Gumbel copula that can capture the extreme negative dependence would be a better fit to the chosen currencies. Examining the null hypothesis that the dependence among the returns of the spot exchange rates can be modelled by the Gaussian, Gumbel, or Clayton copulas, the goodness-of-fit tests confirmed that the Gumbel copula would be a better fit among the three copulas to model the unconditional data series.

Using one of the common factor analysis techniques, the principal component analysis, two common factors were identified for the chosen currencies for all three different samples (i.e., the sample before the crisis occurred, the sample since the crisis occurred, and the full sample that includes at least one full business cycle). Conditional on these common factors, the estimated dependence parameters of the Clayton and Gumbel copulas are found to be similar to each other as shown in Table 9. In other words, the chosen exchange rates exhibited no clear asymmetric dependence, and thus, both the Clayton and Gumbel copulas are not appropriate tools for modelling the joint dependence of the chosen exchange rate returns. These patterns are also evident when comparing the estimated results of the Kendall' Tau, Spearman's Rho, and Tail dependence between the unconditional and two-factor copula models. Furthermore, the goodness-of-fit tests showed that the two-factor Gaussian copula is a good fit for modelling the joint distribution of the chosen currencies. Therefore, the copula correlations obtained from the conditional Gaussian copula are reliable estimates.

Notably, without conditional on the common factors into the copula modelling analysis, the joint dependence among the returns is asymmetric, and thus the unconditional Gumbel copula is a more appropriate tool to model these returns' joint dependence. However, conditional on the common factors, the joint dependence is symmetric, and thus the two-factor Gaussian copula is a better tool. These findings are particularly interesting since they confirm that the common factors play an important role in determining the joint dependence among the exchange rate returns. The common factors can be oil price, global consumer confidence index, or the combination of these factors. When the returns on these factors change, all exchange rate returns will be affected to different degrees depending on how much their representative economies are tied with these factors, and this contributes to the asymmetric joint distributions of the chosen returns. To focus solely on the interactions of the dependence among the currencies themselves, the two-factor Gaussian copula models conditional on the common factors are preferable.

Summing up, the Clayton copula would fit best if negative changes in the chosen exchange rate returns are more highly correlated than positive changes; the Gumbel copula would fit best in the opposite situation. The Gaussian copula fits best if the dependence among the data series is symmetric. The above results analysis indicates that the estimated dependence results are similar under both the conditional Clayton and Gumbel copulas. Combining with the goodness-of-fit tests, the results analysis leads the conclusion that the two-factor Gaussian copula is a good fit for these four exchange rate returns. Malevergne and Sornette (2003) failed to reject the Gaussian copula hypothesis at the $95 \%$ confidence level for more than $50 \%$ of the pairs of currencies over the five-year time interval. In this research, the study is extended from the pairs of currencies to the joint distributions of the chosen four currencies, and found that the symmetric joint dependence among the returns of these currencies can be appropriately modeled with the Gaussian copula hypothesis.

\section{Conclusions}

This paper applies multivariate copula modeling methods in order to study the dependence relationships of daily returns of the four exchange rates: the Canadian Dollar, British Pound, Japanese Yen, and Euro. Conditional on the common factors identified, the copulas are used to estimate the dependence parameters (theta) and their corresponding rank correlation (e.g., Spearman's rho) for two different copulas: Clayton and Gumbel copulas. These two copulas capture the left and right tail dependence, respectively. For the Gaussian copula, we obtained the linear correlation parameters instead of rank correlations to capture the dependence relationships. In the two-step estimation approach, we first obtained empirical CDFs to model the marginal distribution functions, and then we used these CDFs to estimate the dependence parameter in the maximum likelihood function for each copula. For exchange rate returns in our case, the copula modeling method gives more accurate results on the dependence relationships since we are able to utilize this method to capture the nonlinear dependence relationships between non-Gaussian daily returns data of exchange rates. Notably, since the common factors may affect the chosen exchange rates to different degrees and this may contribute to the asymmetric dependence measures, to focus solely 
on the interactions among the exchange rates, we used the copula models conditional on the common factors, which are estimated from principal component analysis.

A few researchers such as Longin and Solnik (2001) found that the stock market exhibit greater left tail dependence, and in other words, the stock markets tend more likely to crash together than go up together. This intrigues us to explore if the foreign exchange markets exhibit similar pattern, and in other words, whether the assumption that the chosen exchange rates have a symmetric dependence structure is consistent with the data. One of the key findings of this paper is that, without conditional on the common factors, the dependence among the chosen currencies is strongly asymmetric and the unconditional Gumbel copula is preferable. In contrast, another key finding is that, conditional on the common factors, the dependence among the chosen currencies is weakly asymmetric and the conditional Gaussian copula modelling hypothesis is more appropriate.

This paper may serve as a brief introduction to the concept of factor copulas and their modeling and estimation methods. These techniques determine more accurate dependent relationships between currencies and better assist the risk assessment, portfolio management, option pricing, and hedging at government, corporate, and individual investor levels. Since 2001, the literature on copulas has grown quickly in the fields of finance and economics. There are several directions for future research and applications on conditional copula modeling that we would like to point out here. First, it may be of interest to apply the factor copula modelling method to study dependence relationships among the foreign exchange markets and the financial stock markets. Factor copula modeling could also be applied to risk management and asset pricing. In addition, the copulas we used and other copulas could be extended to model the joint distribution of returns, volume, and duration between transactions for foreign exchange rates.

\section{References}

Andersen, L., \& Sidenius, J. (2004). Extensions to the Gaussian copula: random recovery and random factor loadings. Journal of Credit Risk. 1(1), 29-70.

Granger, C. W. J., Terasvrta, T., \& Patton, A. J. (2006). Common Factors in Conditional Distributions for Bivariate Time Series. Journal of Econometrics, 132(1), 43-57. http://dx.doi.org/10.1016/j.jeconom.2005.01.022

Hurd, M., Salmon, M., \& Schleicher, C. (2007). Using copulas to construct bivariate foreign exchange distributions with an application to the sterling exchange rate index, Bank of England working papers, 334, Bank of England.

$\mathrm{Hu}$, L. (2006). Dependence patterns across financial markets: a mixed copula approach. Applied Financial Economics, 16(10), 717-729. http://dx.doi.org/10.1080/09603100500426515

Hull, J. (2008). Options, futures, and other derivatives, $\left(7^{\text {th }}\right.$ ed.) New Jersey: Pearson Education, (Chapter 22).

Jolliffe, I. T. (1986). Principal Components Analysis, ( $1^{\text {st }}$ ed.) New York: Springer.

Longin, F., \& Solnik, B. (2001). Extreme correlation of international equity markets. Journal of Finance, 56(2), 649-676. http://dx.doi.org/10.1111/0022-1082.00340

Malevergne, Y., \& Sornette, D. (2003). Testing the Gaussian copula hypothesis for financial assets dependence. Quantitative Finance, 3(4), 231-250. http://dx.doi.org/10.1088/1469-7688/3/4/301

Nelson, R. B. (1999). An Introduction to Copulas, New York: Springer, (chapter 5).

Patton, A. (2006). Modelling asymmetric exchange rate dependence. International Economic Review, 47(2), 527-556. http://dx.doi.org/10.1111/j.1468-2354.2006.00387.x

Patton, A. (2009). Copula-based models for financial time series, in: T.G. Andersen, R. A. Davies, J. P. Kreiss, and T. Mikosch, editions. Handbook of Financial Times Series, Berlin: Springer.

Schmidt, T. (2006). An Infinite Factor Model for Credit Risk. International Journal of Theoretical and Applied Finance, 9(1), 43-68. http://dx.doi.org/10.1142/S0219024906003482

Sklar, A. (1959). Fonctions de répartition à n dimensions et leurs marges. Publications de l'Institut de Statistique de L'Université de Paris, 8, 229-31.

Trivedi, P., \& Zimmer, D. (2005). Copula modeling: an introduction for practitioners. Foundations and Trends in Econometrics, 1(1), 1-111. http://dx.doi.org/10.1561/0800000005 
Table 5a. Factor Loadings of the Transformed Normal Variables

\begin{tabular}{cccccccccc}
\hline & \multicolumn{3}{c}{ Full Sample } & \multicolumn{3}{c}{ Pre-Crisis Sample } & \multicolumn{3}{c}{ Post-Crisis Sample } \\
\hline & Factor 1 & Factor 2 & Uniqueness & Factor 1 & Factor 2 & Uniqueness & Factor 1 & Factor 2 & Uniqueness \\
\hline rcad & 0.46 & -0.66 & 0.35 & 0.03 & 0.97 & 0.051 & 0.66 & -0.35 & 0.448 \\
reur & 0.89 & 0.04 & 0.21 & 0.88 & 0.19 & 0.195 & 0.86 & 0.14 & 0.236 \\
rjpy & 0.86 & 0.03 & 0.26 & 0.88 & 0.11 & 0.215 & 0.84 & 0.01 & 0.298 \\
rgdp & 0.33 & 0.79 & 0.27 & 0.66 & -0.19 & 0.535 & 0.02 & 0.96 & 0.081 \\
\hline
\end{tabular}

Table 5b. Proportions Explained by the Common Factors of the Transformed Variables

\begin{tabular}{cccc}
\hline & \multicolumn{3}{c}{ Proportion of Variance } \\
\hline Proportion Explained by & Full Sample & Pre-Crisis Sample & Post-crisis sample \\
\hline Factor 1 & 0.46 & 0.49 & 0.47 \\
Factor 2 & 0.27 & 0.26 & 0.26 \\
Cumulative Proportions & 0.73 & 0.75 & 0.73 \\
\hline
\end{tabular}

Table 6. Unconditional Gaussian Copula Results

\begin{tabular}{lccc}
\hline & Full Sample & Pre-Crisis Sample & Post-Crisis Sample \\
\hline Rho (rc, rj) & 0.266391 & 0.157107 & 0.398597 \\
Rho (rc, rg) & 0.212978 & 0.083562 & 0.354494 \\
Rho (rc, re) & -0.06569 & 0.003878 & -0.14168 \\
Rho (rg, rj) & 0.683859 & 0.761216 & 0.625544 \\
Rho (re, rj) & 0.223964 & 0.371452 & 0.072583 \\
Rho (re, rg) & 0.165226 & 0.363254 & -0.03366 \\
\hline
\end{tabular}

Table 7. Factor Gaussian Copula Results

\begin{tabular}{lccc}
\hline & Full Sample & Pre-Crisis Sample & Post-Crisis Sample \\
\hline Rho (rc, rj) & 0.751957 & 0.264077 & 0.952702 \\
Rho (rc, rg) & -0.73271 & -0.42395 & -0.61651 \\
Rho (rc, re) & 0.470419 & 0.227673 & 0.714544 \\
Rho (rg, rj) & -0.12428 & 0.652986 & -0.46903 \\
Rho (re, rj) & 0.910232 & 0.913827 & 0.866368 \\
Rho (re, rg) & 0.153417 & 0.713231 & -0.04502 \\
\hline
\end{tabular}

Table 8a. Factor Loadings of the Exchange Rate Returns

\begin{tabular}{cccccccccc}
\hline & \multicolumn{3}{c}{ Full Sample } & \multicolumn{3}{c}{ Pre-Crisis Sample } & \multicolumn{3}{c}{ Post-Crisis Sample } \\
\hline & Factor 1 & Factor 2 & Uniqueness & Factor 1 & Factor 2 & Uniqueness & Factor 1 & Factor 2 & Uniqueness \\
\hline rcad & 0.56 & -0.55 & 0.38 & 0.04 & 0.97 & 0.051 & 0.67 & -0.39 & 0.398 \\
reur & 0.89 & 0.14 & 0.18 & 0.88 & 0.2 & 0.188 & 0.88 & 0.18 & 0.19 \\
rjpy & 0.19 & 0.89 & 0.18 & 0.65 & -0.19 & 0.539 & 0.02 & 0.96 & 0.086 \\
rgdp & 0.86 & 0.03 & 0.25 & 0.88 & 0.11 & 0.217 & 0.85 & -0.02 & 0.273 \\
\hline
\end{tabular}


Table 8b. Proportion Explained by the Common Factors of the Exchange Rate Returns

\begin{tabular}{cccc}
\hline & \multicolumn{3}{c}{ Proportion of Variance } \\
\hline Proportion Explained by & Full Sample & Pre-Crisis Sample & Post-crisis sample \\
\hline Factor 1 & 0.48 & 0.49 & 0.49 \\
Factor 2 & 0.28 & 0.26 & 0.27 \\
Cumulative Proportions & 0.75 & 0.75 & 0.76 \\
\hline
\end{tabular}

Table 9. Unconditional and Factor Clayton \& Gumbel Copula Estimation Results

\begin{tabular}{ccccc}
\hline Copula & & Full Sample & Pre-Crisis Sample & Post-Crisis Sample \\
\hline \multirow{4}{*}{ Unconditional } & Dependence Parameter & 0.5182035 & 0.6735244 & 0.4390656 \\
Clayton Copula & Kendall's Tau & 0.205783 & 0.2519238 & 0.1800138 \\
& Spearman's Rho & 0.3038211 & 0.368643 & 0.2669235 \\
& Tail Index & {$[0.26247,0]$} & {$[0.3573161,0]$} & {$[0.2062457,0]$} \\
& Dependence Parameter & 1.266502 & 1.337486 & 1.237634 \\
Unconditional & Kendall's Tau & 0.2104238 & 0.2523284 & 0.1920068 \\
Gumbel Copula & Spearman's Rho & 0.3104698 & 0.3683127 & 0.2841702 \\
& Tail Index & {$[0,0.27143]$} & {$[0,0.3209192]$} & {$[0,0.2492256]$} \\
& Dependence Parameter & 4.197289 & 3.872766 & 3.712977 \\
Factor & Kendall's Tau & 0.6770352 & 0.6588136 & 0.6505415 \\
Clayton Copula & Spearman's Rho & 0.8551584 & 0.8399345 & 0.8328992 \\
& Tail Index & {$[0.847617,0]$} & {$[0.8357017,0]$} & {$[0.8301302,0]$} \\
& Dependence Parameter & 3.595267 & 3.685626 & 3.289485 \\
Factor & Kendall's Tau & 0.7218565 & 0.7286757 & 0.696001 \\
Gumbel Copula & Spearman's Rho & 0.8923946 & 0.8973188 & 0.8730768 \\
& Tail Index & {$[0,0.78736]$} & {$[0,0.7930848]$} & {$[0,0.7654383]$} \\
\hline
\end{tabular}

